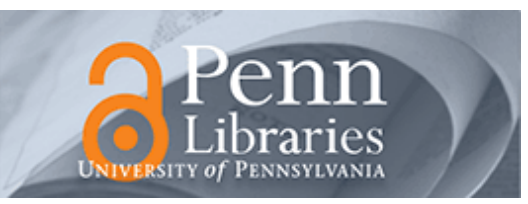

University of Pennsylvania

ScholarlyCommons

September 1994

\title{
Theoretical Study of Variation of Propagation Constant in a Cylindrical Waveguide Due to Chirality: Chiro-Phase Shifting
}

\author{
Mamdouh M. I. Saadoun \\ Saudi Consolidated Electric Company \\ Nader Engheta \\ University of Pennsylvania, engheta@ee.upenn.edu
}

Follow this and additional works at: https://repository.upenn.edu/ese_papers

\section{Recommended Citation \\ Mamdouh M. I. Saadoun and Nader Engheta, "Theoretical Study of Variation of Propagation Constant in a Cylindrical Waveguide Due to Chirality: Chiro-Phase Shifting", . September 1994.}

Copyright 1994 IEEE. Reprinted from IEEE Transactions on Microwave Theory and Techniques, Volume 42, Issue 9, September 1994, pages 1690-1694.

This material is posted here with permission of the IEEE. Such permission of the IEEE does not in any way imply IEEE endorsement of any of the University of Pennsylvania's products or services. Internal or personal use of this material is permitted. However, permission to reprint/republish this material for advertising or promotional purposes or for creating new collective works for resale or redistribution must be obtained from the IEEE by writing to pubs-permissions@ieee.org. By choosing to view this document, you agree to all provisions of the copyright laws protecting it.

This paper is posted at ScholarlyCommons. https://repository.upenn.edu/ese_papers/255

For more information, please contact repository@pobox.upenn.edu. 


\title{
Theoretical Study of Variation of Propagation Constant in a Cylindrical Waveguide Due to Chirality: Chiro-Phase Shifting
}

\author{
Abstract \\ We discuss a conceptual idea for a reciprocal phase shifting using chiral materials. Such phase shifters, \\ which can be called chiro-phase shifers, may, in principle, consist of a general cylindrical waveguide in \\ which a thin chiral rod is inserted longitudinally. A standard commonly used perturbation technique is \\ utilized to analyze theoretically the effects of a chiral rod's parameters (and in particular its chirality \\ admittance) on propagation constants of guided modes in such waveguides. A simple expression relating \\ the relative change of propagation constants to other relevant quantities such as polarization \\ characteristics of unperturbed guided modes, material parameters of chiral rod, and location of the rod is \\ presented, and the case of a circular metallic chiro-phase shifter is discussed in some detail. Physical \\ insights into the results are also provided.

\section{Comments} \\ Copyright 1994 IEEE. Reprinted from IEEE Transactions on Microwave Theory and Techniques, Volume 42, \\ Issue 9, September 1994, pages 1690-1694. \\ This material is posted here with permission of the IEEE. Such permission of the IEEE does not in any way \\ imply IEEE endorsement of any of the University of Pennsylvania's products or services. Internal or \\ personal use of this material is permitted. However, permission to reprint/republish this material for \\ advertising or promotional purposes or for creating new collective works for resale or redistribution must \\ be obtained from the IEEE by writing to pubs-permissions@ieee.org. By choosing to view this document, \\ you agree to all provisions of the copyright laws protecting it.
}




\title{
Theoretical Study of Variation of Propagation Constant in a Cylindrical Waveguide Due to Chirality: Chiro-Phase Shifting
}

\author{
Mamdouh M. I. Saadoun and Nader Engheta
}

\begin{abstract}
We discuss a conceptual idea for a reciprocal phase shifting using chiral materials. Such phase shifters, which can be called chiro-phase shifters, may, in principle, consist of a general cylindrical waveguide in which a thin chiral rod is inserted longitudinally. A standard commonly used perturbation technique is utilized to analyze theoretically the effects of a chiral rod's parameters (and in particular its chirality admittance) on propagation constants of guided modes in such waveguides. A simple expression relating the relative change of propagation constants to other relevant quantities such as polarization characteristics of unperturbed guided modes, material parameters of chiral rod, and location of the rod is presented, and the case of a circular metallic chiro-phase shifter is discussed in some detail. Physical insights into the results are also provided.
\end{abstract}

\section{INTRODUCTION}

$\mathbf{T}$ HE similarities between chiral media, which in the optical regime are also known as media with natural optical activity [1], and magnetically biased ferrites have been addressed and studied since the early days of the introduction of the concept of chirality in electromagnetics and optics. However, to date, relatively less has been done to explore the possibility of using chiral materials instead of (or combined with) magnetically biased ferrites in the design of novel devices and components. Here, an idea for a reciprocal phase shifter whose principles of operation lie in the properties of chiral materials is discussed. Unlike magnetically biased ferrite devices, which are generally nonreciprocal [2]-[4], this proposed device can conceptually be reciprocal owing to the property of reciprocity in isotropic chiral media [5]-[7]. This may be of interest in certain applications of phase shifters where reciprocity is desired. We have proposed elsewhere [8] another conceptual idea for a reciprocal phase shifter based on a certain class of bianisotropic media, which we introduced recently and called the pseudochiral $\Omega$-medium.

An isotropic chiral material is a medium whose microstructures lack mirror symmetry leading to the property of handedness. Such a property is reflected in the constitutive relations of

Manuscript received May 22, 1992; revised October 27, 1993. This work was supported in part by the National Science Foundation under Presidential Young Investigator Grant ECS-8957434, and in part by the Complex Media Laboratory, Moore School of Electrical Engineering, University of Pennsylvania.

M. M. I. Saadoun was with the Moore School of Electrical Engineering, University of Pennsylvania, Philadelphia, PA 19104 USA. He is now with the Information Center, Central Region, Saudi Consolidated Electric Company, Riyadh, 11411, Saudi Arabia.

N. Engheta is with the Moore School of Electrical Engineering, University of Pennsylvania, Philadelphia, PA 10104 USA.

IEEE Log Number 9404081. the medium which can take the form $\boldsymbol{D}=\epsilon_{c} \boldsymbol{E}+i \xi_{c} \boldsymbol{B}$ and $\boldsymbol{H}=$ $i \xi_{c} \boldsymbol{E}+\left(1 / \mu_{c}\right) \boldsymbol{B}$. The additional parameter $\xi_{c}$ is the chirality admittance describing the handedness of the medium, and $\epsilon_{c}$ and $\mu_{c}$ are the permittivity and permeability of the medium, respectively [9]. The eigenmodes of propagation in chiral media are shown to be right-circularly polarized (RCP) and left-circularly polarized (LCP) plane waves with two differing propagation constants $k_{ \pm}=\omega\left[ \pm \mu_{c} \xi_{c}+\sqrt{\epsilon_{c} \mu_{c}+\mu_{c}^{2} \xi_{c}^{2}}\right]$, where the plus and minus signs refer to the RCP and LCP eigenmodes, respectively [5]-[7]. It is also well known that in the case of magnetically biased ferrites, the eigenmodes of propagation along the direction of the biasing magnetic field are, also, RCP and LCP plane waves with two unequal propagation constants [10], [11]. It is in this sense that a chiral medium is similar to a magnetically biased ferrite material. However, it is well known that while the former is a reciprocal medium, the latter is not. Furthermore, in isotropic chiral media, the two wavenumbers $k_{ \pm}$shown above are independent of the direction of propagation, whereas magnetically biased ferrites are intrinsically anisotropic, and their wavenumbers of eigenmodes are indeed direction-dependent. Since magnetically biased ferrites have been extensively utilized in the design of a variety of microwave devices and components [2]-[4], similarities between such materials and chiral media have motivated us to investigate theoretically the possibility of utilizing chiral materials in the design of devices which would be counterparts to ferrite devices. Phase shifters, in particular, are good candidates for this study of chiral-based devices since they are not functionally required to always be nonreciprocal. As in the case of ferrite phase shifters where a thin ferrite slab inserted in a cylindrical waveguide affects the propagation constant of the guide, here we discuss theoretically similar effects when a thin rod of chiral material is to be inserted in a waveguide. Such a device may be called the chiro-phase shifter.

In the following section, we will review the variation of propagation constant due to the insertion of a thin chiral rod in a generic waveguide (metallic or open waveguide) with arbitrary cross section. In Section III, as an illustrative example, we will analyze a special device configuration for a chiro-phase shifter.

\section{VARIATION OF THE GUIDE WAVENUMBERDUE TO INSERTION OF A THIN CHIRAL ROD}

Fig. 1 presents the geometry of the present problem where a cylindrical waveguide with arbitrary cross-sectional shape 
is loaded with a longitudinal thin rod of chiral material. The waveguide can have a perfectly conducting boundary or it can be an open waveguide such as a dielectric waveguide supporting a guided surface wave. The waveguide, before insertion of the chiral rod, is filled with a material with permeability $\mu$ and permittivity $\epsilon$. The lossless chiral rod, with material parameters $\epsilon_{c}, \mu_{c}$, and $\xi_{c}$, is assumed to have small transverse dimensions compared with the waveguide dimensions and the free-space wavelength of the propagating guided electromagnetic wave. The exact shape of the rod's cross section is not important, but it is assumed to be smooth and not to have sharp corners or edges. In general, the rod can be placed anywhere in the waveguide with its axis parallel to the waveguide axis. ${ }^{1}$ Since the exact solutions for guided modes in this loaded waveguide are obtainable only for a few special geometries, here for the general geometry of the waveguides and chiral rods, the standard perturbation technique must be used to study the effects of the thin chiral rod on the guide propagation constant. This perturbation technique has been previously used for cavity resonators wherein a small bianisotropic or biisotropic sample has been inserted. For an excellent description of this technique, the reader is referred to [12], [13]. For the perturbation theory for waveguides, the reader is referred to the work of Waldron [10] and Viitanen and Lindell [14]. In [14], Viitanen and Lindell have done an excellent analysis for perturbation theory in a corrugated waveguide with an inserted biisotropic rod. Here, to explore the role of chiral material (instead of magnetically biased ferrite) in the possible design of a phase shifter, this commonly used perturbation technique [10] for a waveguide is used for a general cylindrical waveguide (metallic or open waveguide) within which a thin chiral rod is inserted. It must be noted that this perturbation technique [10], [12]-[14], which can be applicable to many problems of this sort, is used where insertion of the rod does not alter appreciably the field distributions in the waveguide. Let the unperturbed electric field of the guided mode before insertion of the rod be denoted by $\boldsymbol{E}$ and be given by $\boldsymbol{E}=\boldsymbol{E}^{(0)}(x, y) e^{i \beta z} e^{-i \omega t}$, and the perturbed electric field of the guided mode after the rod is inserted be shown by $\boldsymbol{E}^{\prime}=\left[\boldsymbol{E}^{(0)}(x, y)+\boldsymbol{E}^{(1)}(x, y)\right]$. $e^{i(\beta+\delta \beta) z} e^{-i \omega t}$. Following a procedure similar to the one described in [10], [12], [13], one can get the following expression for the relative perturbation of the propagation constant, i.e., $\delta \beta / \beta$ in (1), shown at the bottom of the page, where the asterisk denotes complex conjugation, $\boldsymbol{E}^{(0)}$, $D^{(0)}, \boldsymbol{H}^{(0)}$, and $\boldsymbol{B}^{(0)}$ are the unperturbed fields, and $\boldsymbol{E}^{(1)}$ $\boldsymbol{E}^{(1)}, \boldsymbol{E}^{(1)}$, and $\boldsymbol{E}^{(1)}$ are their incremental perturbations, respectively. For a derivation of an expression similar to (1),

\footnotetext{
${ }^{1}$ As will be shown later, clearly it would not be preferred to locate the rod in regions of vanishing unperturbed fields.
}

the reader is referred to [10], [12], [13]. ${ }^{2}$ It must be noted that, like in [14], here in (1) the quantity of particular interest is $\delta \beta / \beta$, whereas in the perturbation analysis given in [12], [13], the relative shift in resonance frequency, i.e., $\delta \omega / \omega$, is of importance. As in [10], [12]-[14], the integration in the denominator of (1) is carried out over the whole cross section $S_{0}$ of the waveguide, while that in the numerator is performed over the cross section of the perturbing rod $S_{1}$ only. This means that it is assumed that the fields are perturbed mainly within the perturbing rod itself. To evaluate $\delta \beta / \beta$, first we need to know the unperturbed fields of the guided modes, which are usually given, and then we must estimate the fields' incremental perturbations, $E^{(1)}, H^{(1)}, D^{(1)}$, and $B^{(1)}$. To that end, a first-order Born-type approximation is used, and the thin chiral rod is treated as a small-size "scatterer." Furthermore, to use such an approximation, it is also assumed that variation of material parameters (before and after insertion of the chiral rod) is small in magnitude, i.e., $\left|\mu_{c}-\mu\right| \ll \mu$, $\left|\epsilon_{c}-\epsilon\right| \ll \epsilon$, and $\xi_{c} \ll \sqrt{\epsilon / \mu}$ [10], [15]. In this approximation, the incident fields, which are actually the unperturbed guided mode's fields, are assumed to remain unchanged (in lowest order approximation) within the chiral rod and induce electric polarization density $\boldsymbol{P}$ and magnetization $\boldsymbol{M}$ within the chiral rod. Thus, the rod can be replaced by these induced $\boldsymbol{P}$ and $\boldsymbol{M}$. It is worth noting that in order to find the fields inside the rod, one could also use other techniques, such as the quasi-static analysis when the chiral rod has a symmetric shape such as a circular cylindrical shape. In that case, one would need to use the polarizability dyadics. For small bianisotropic spheres, the polarizability dyadics have been studied extensively by Lakhtakia [16] and for biisotropic elliposoids by Sihvola [17], and they have been used in the perturbation analysis for the cavity resonators [12], [13]. Here, however, since the crosssectional shape of the chiral rod has been assumed to be arbitrary in this section, the Born-type approximation is being used, although we realize that this approximation will provide an approximate (not exact) expression for the fields inside the rod. Since polarization and magnetization sources contribute to the displacement vector $D$ and magnetic intensity vectors $\boldsymbol{H}$, respectively, it seems to be reasonable to consider that, in this approximation, the electric field intensity $\boldsymbol{E}$ and the magnetic induction $\boldsymbol{B}$ remain unchanged within the chiral rod, while the displacement vector $D$ and magnetic intensity vectors $\boldsymbol{H}$ are perturbed. ${ }^{3}$ Therefore, we have $\boldsymbol{E}^{(1)} \cong 0$ and

\footnotetext{
${ }^{2}$ It must be noted that unlike (1), here, the analogous expression in [10] does not contain any complex conjugation. The use of complex conjugates in expression (1) here is advantageous because it allows the direct substitution of waveguide field expressions in the equation without any modification. This is not the case, however, for the similar expression given in [10] where an additional "rule of thumb," suggested by the author of [10], has to be applied prior to substitution.

${ }^{3}$ As a matter of fact, one could have assumed any pair of fields $(E, H)$, $(\boldsymbol{E}, \boldsymbol{B}),(\boldsymbol{D}, \boldsymbol{H})$, or $(\boldsymbol{D}, \boldsymbol{B})$ to remain unperturbed within the chiral rod
}

$$
\frac{\delta \beta}{\beta}=-\frac{\omega}{\beta} \frac{d \beta}{d \omega} \frac{\int_{S_{1}}\left\{\boldsymbol{E}^{(1)} \cdot \boldsymbol{D}^{(0)^{*}}-\boldsymbol{E}^{(0)^{*}} \cdot \boldsymbol{D}^{(1)}+\boldsymbol{H}^{(1)} \cdot \boldsymbol{B}^{(0)^{*}}-\boldsymbol{H}^{(0)^{*}} \cdot \boldsymbol{B}^{(1)}\right\} d S_{1}}{\int_{S_{0}}\left\{\boldsymbol{E}^{(0)} \cdot \boldsymbol{D}^{(0)^{*}}+\boldsymbol{H}^{(0)^{*}} \cdot B^{(0)}\right\} d S_{0}}
$$


$B^{(1)} \cong 0$. From the above equation and the constitutive relations in chiral media, the following relations for the fields' incremental perturbations are $\boldsymbol{D}^{(1)} \cong\left(\epsilon_{c}-\epsilon\right) \boldsymbol{E}^{(0)}+i \xi_{c} \boldsymbol{B}^{(0)}$ and $\boldsymbol{H}^{(1)} \cong\left(\left(1 / \mu_{c}\right)-(1 / \mu)\right) \boldsymbol{B}^{(0)}+i \xi_{c} \boldsymbol{E}^{(0)}$. Having obtained the unperturbed and incremental perturbations of the field, one can find $\delta \beta / \beta$ from (1) as given below:

$$
\begin{aligned}
\frac{\delta \beta}{\beta}=-f(\omega)\left\{\frac{\epsilon_{c}-\epsilon}{4}\left|\boldsymbol{E}^{(0)}\right|^{2}-\frac{1}{4}\left(\frac{1}{\mu_{c}}-\frac{1}{\mu}\right)\left|\boldsymbol{B}^{(0)}\right|^{2}\right. \\
\left.+\frac{\xi_{c}}{2} \operatorname{Im}\left(\boldsymbol{E}^{(0)} \cdot \boldsymbol{B}^{(0) *}\right)\right\}\left.\right|_{\rho \rightarrow \mathrm{rod}^{\prime} \text { s center }}
\end{aligned}
$$

where $f(\omega) \equiv-4\left(\omega S_{1} / \beta\right)(d \beta / d \omega)\left[\int_{S_{0}}\left\{\boldsymbol{E}^{(0)} \cdot \boldsymbol{D}^{(0) *}+\boldsymbol{H}^{(0) *}\right.\right.$. $\left.\left.B^{(0)}\right\} d S_{0}\right]^{-1}$ and the symbol $\rho \rightarrow$ rod's center means that the expressions in curly brackets are evaluated at the center of the rod. ${ }^{4}$ The function $f(\omega)$ is independent of the material parameters of the perturbing rod, and depends only on the waveguide geometry, the unperturbed waveguide fields, and the cross-sectional area of the rod. The degree of accuracy of this perturbation method depends on the relative difference of material parameters of the chiral rod and the waveguide material. As mentioned earlier, such relative differences are to be small, i.e., $\left|\mu_{c}-\mu\right| \ll \mu,\left|\epsilon_{c}-\epsilon\right| \ll \epsilon$, and $\xi_{c} \ll \sqrt{\epsilon / \mu}$. It must be noted, however, that as an alternative, numerical techniques such as the finite-element method can be used to obtain the propagation constants in chiral loaded waveguides [18]. Equation (2) shows that the chirality of the rod has a direct effect (to the first order) on propagation constant $\beta$ when the imaginary part of the scalar product $\left(\boldsymbol{E}^{(0)} \cdot \boldsymbol{B}^{(0) *}\right)$ of the unperturbed fields has a nonvanishing value. This can happen when $B^{(0)}$ has a component along $\boldsymbol{E}^{(0)}$ with some nonzero phase difference. For example, if $\boldsymbol{E}^{(0)}$ and $\boldsymbol{B}^{(0)}$ are elliptically polarized fields, $\operatorname{Im}\left(\boldsymbol{E}^{(0)} \cdot \boldsymbol{B}^{(0) *}\right)$ may be nonzero depending on the orientation of the polarization ellipses of these two fields. If $\boldsymbol{E}^{(0)}$ and $\boldsymbol{B}^{(0)}$ are in phase and linearly polarized, $\operatorname{Im}\left(\boldsymbol{E}^{(0)} \cdot \boldsymbol{B}^{(0) *}\right)$ will be zero. Clearly, the perturbation of the propagation constant due to chirality is strongest for circularly polarized fields. For the case of linearly polarized fields in waveguides, and for the case of perturbation of modes of corrugated waveguides due to the biisotropic rod, the reader is referred to [8] and [14], respectively. The importance of the imaginary part of $\left(\boldsymbol{E}^{(0)} \cdot \boldsymbol{B}^{(0) *}\right)$ in the shift of resonance frequency in cavity resonators was discussed in [13].

From a microscopic point of view, one can visualize the chiral medium as a collection of small helices of certain handedness. The incident (unperturbed) electric field polarizes the stem of the helix. Since the field is time varying, a current flows in the stem, and consequently in the loop portion of the

in this approximation, and then obtained incremental perturbations to the other remaining fields. It can be shown that the results, however, would have remained the same to within the first order in the perturbation parameter $\left(\mu_{c}-\mu\right),\left(\epsilon_{c}-\epsilon\right)$, and $\xi_{c}$.

${ }^{4}$ Strictly speaking, the expressions in the curly brackets should be evaluated at all points on the rod's cross section in order to obtain the value of the integral in the numerator of (1). However, since the rod is taken to be thin, it can be assumed that the integrand of this integral does not vary appreciably over the rod's cross section, and therefore its value can be obtained at one point, namely, the center of the $\operatorname{rod} \rho \rightarrow$ rod's center. This value should then be multiplied by the cross-sectional area of the rod to get the integral. This area is lumped into the function $f(\omega)$.

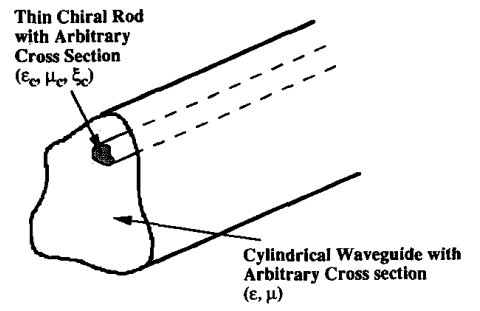

Fig. 1. A cylindrical waveguide with arbitrary cross section loaded longitudinally with a thin rod of lossless chiral material with parameters $\epsilon_{c}, \mu_{c}$, and $\xi_{c}$. The cross-sectional shape of the thin rod can also be arbitrary. Aside from the chiral rod, the waveguide is filled with a lossless material whose permittivity and permeability are $\epsilon$ and $\mu$, respectively.

helix. This current, which has a $90^{\circ}$ phase difference with the inducing electric field, provides a magnetic dipole moment. This magnetic moment, which is in phase with the induced current, is therefore $90^{\circ}$ out of phase with the inducing electric field, and is mainly parallel to the stem of the helix. Hence, the incident electric field induces a magnetization parallel to the electric field and $90^{\circ}$ out of phase with it. If the incident unperturbed wave has a magnetic field component parallel to the inducing electric field with a nonzero phase difference with it, there will be some interaction between the electrically induced magnetization and the incident magnetic field. A similar argument can be addressed for the interaction between the magnetically induced electric dipole moments and the incident electric field. The above discussion provides some intuitive insight into (2). Here, it is reviewed that the chirality of the thin rod can, in principle, affect the propagation constant $\beta$, and consequently the phase $\varphi$ of a signal propagating through the guide. Therefore, phase shifting can be achieved in such a device. This phase shifter may be called the chiro-phase shifter. It is worth noting that in order to use chirality as a mechanism for achieving variable shift on the phase of propagating signals in a waveguide, one should find ways by which the chirality can be varied in real time through the variation of other quantities such as biasing fields. Such variation and control of chirality, particularly in optically active materials and in chiral liquid crystals, is presently under study. Combining the effect of chirality and Faraday rotation was also theoretically studied to explore possible ways to control chirality [19]. Moreover, a change of the chiral rod's loction may also lead to a change in propagation constant $\beta$, and hence in phase $\varphi$. Thus, this idea can be a basis for a device in sensing displacement and flow speed. In the following section, as an example, we apply the results of the perturbation technique to a specific case of a circular metallic chiro-phase shifter where a circular cylindrical metallic waveguide is loaded with a thin chiral rod located at an arbitrary position.

\section{AN EXAMPle: A CiRCUlar ChIRO-PHASE SHIFTER}

Consider a device geometry in which a circular cylindrical waveguide with metallic walls is loaded longitudinally with a thin rod of chiral material located at some arbitrary position. Apart from the chiral rod, the waveguide is assumed to be 
filled with a homogeneous isotropic dielectric of permittivity $\epsilon$ and permeability $\mu$. The material parameters of the rod are $\epsilon_{c}, \mu_{c}, \xi_{c}$. As mentioned earlier, it is also assumed that $\left|\mu_{c}-\mu\right| \ll \mu,\left|\epsilon_{c}-\epsilon\right| \ll \epsilon$, and $\xi_{c} \ll \sqrt{\epsilon / \mu}$. The radius of the $\operatorname{rod} d$ is assumed to be much smaller than the radius of the waveguide $R$. These assumptions are required to justify the use of perturbation techniques in analyzing this problem. The exact solutions for guided electromagnetic waves in such loaded waveguides in general (with arbitrary location of the chiral rod) appear to be untractable in closed form. For special cases, such as the case where the chiral rod is circular and located at the center of the circular waveguide, the closed form solutions are obtainable. This problem has been studied by Monte and Uslenghi [20]. Here, however, we consider the general case where the rod is located longitudinally at some arbitrary location other than the waveguide's axis, and as a result, we need to use the perturbation technique described in the previous section. It is well known that the field components of the dominant $\mathrm{TE}_{11}$ mode of this unperturbed waveguide (with $E_{z}^{(0)}=0$ ) can be written as [11]

$$
\begin{gathered}
H_{z}^{(0)}=J_{1}\left(\frac{x_{11}^{\prime}}{R} \rho\right)\left\{\begin{array}{l}
C_{1} \cos \phi \\
C_{2} \sin \phi
\end{array} \quad e^{i \beta z} e^{-i \omega t}\right. \\
H_{\rho}^{(0)}=-E_{\phi}^{(0)} / Z_{\mathrm{TE}_{11}} \\
=\frac{i \beta}{\left(\left(x_{11}^{\prime} / R\right)\right)} J_{1}^{\prime}\left(\frac{x_{11}^{\prime}}{R} \rho\right)\left\{\begin{array}{l}
C_{1} \cos \phi \\
C_{2} \sin \phi
\end{array} e^{i \beta z} e^{-i \omega t}\right.
\end{gathered}
$$

$$
\begin{aligned}
H_{\phi}^{(0)} & =E_{\rho}^{(0)} / Z_{\mathrm{TE}_{11}} \\
& =\frac{i \beta}{\left(\left(x_{11}^{\prime} / R\right)\right)^{2} \rho} J_{1}\left(\frac{x_{11}^{\prime}}{R} \rho\right)\left\{\begin{array}{r}
-C_{1} \sin \phi \\
C_{2} \cos \phi
\end{array} \quad e^{i \beta z} e^{-i \omega t}\right.
\end{aligned}
$$

where $Z_{\mathrm{TE}_{11}}(=\omega \mu / \beta)$ is the transverse waveguide impedance for the $\mathrm{TE}_{11}$ mode, $\beta\left(=\sqrt{\omega^{2} \mu \epsilon-\left(x_{11}^{\prime} / R\right)^{2}}\right)$ is the unperturbed guide propagation constant, $x_{11}^{\prime}$ is the first root of the derivative of the first-order Bessel function of the first kind, i.e., the first root of $d J_{1}(x) / d x=0$, $(\rho, \varphi, z)$ are used as cylindrical coordinate systems for the circular waveguide, and $C_{1}$ and $C_{2}$ are two arbitrary complex constants. By properly choosing the two constants $C_{1}$ and $C_{2}$, one can obtain a desired polarization for the transverse fields at the center (or any other given location) of the guide's cross section. For instance, $C_{1}$ and $C_{2}$ can be chosen to be $C_{1}=C$ and $C_{2}=i p C$ where $C$ is an arbitrary constant and $p$ is a real constant. Thus, one can write

$$
\begin{gathered}
H_{z}^{(0)}=C J_{1}\left(\frac{x_{11}^{\prime}}{R} \rho\right)[\cos \phi+i p \sin \phi] e^{i \beta z} e^{-i \omega t} \\
H_{\rho}^{(0)}=C \frac{i \beta}{\left(\left(x_{11}^{\prime} / R\right)\right)} J_{1}^{\prime}\left(\frac{x_{11}^{\prime}}{R} \rho\right)[\cos \phi+i p \sin \phi] e^{i \beta z} e^{-i \omega t} \\
H_{\phi}^{(0)}=C \frac{i \beta}{\left(\left(x_{11}^{\prime} / R\right)\right)^{2} \rho} J_{1}\left(\frac{x_{11}^{\prime}}{R} \rho\right)[-\sin \phi+i p \cos \phi] \\
\cdot e^{i \beta z} e^{-i \omega t} .
\end{gathered}
$$

The electric field can be obtained in a similar way. From (4), we can clearly see that the transverse electric and magnetic fields at the center of the waveguide are, in general, elliptically polarized. In fact, the transverse magnetic field is given by $\left.\boldsymbol{H}_{t}^{(0)}\right|_{\rho=0}=(C / 2)\left(i \beta \boldsymbol{R} / x_{11}^{\prime}\right)\left[\boldsymbol{e}_{x}+i p \boldsymbol{e}_{y}\right] e^{i \beta z} e^{-i \omega t}$. Here, $\boldsymbol{e}_{x}$ and $e_{y}$ are unit vectors along the $x$ and $y$ axes of a Cartesian coordinate system, and the parameter $p$ describes the axial ratio of the ellipse of polarization of the field at the center of the waveguide. For positive (negative) values of $p$, the field is right (left) elliptically polarized. For $p$ being zero or infinity, the field is linearly polarized. For $p$ being $+1(-1)$, the field is right-circularly polarized RCP (left-circularly polarized LCP). When the location of the chiral rod is given in terms of $\rho_{0}$ and $\varphi_{0}$, the unperturbed field quantities can be obtained from (3) and (4), and the $\mathrm{n}$ the value of $\delta \beta / \beta$ can be evaluated from (2). As can be seen from (2), the relative change in $\beta$ is due to three terms: the first and second terms are the effects of variation of the permittivity and permeability due to insertion of the chiral rod, while the third term describes the effect of the chirality of the rod. Assuming $Z_{\mathrm{TE}_{11}}$ to be real (which requires the unperturbed waveguide to be lossless and the unperturbed mode to be propagating), this third term can be explicitly written at the location of the chiral rod, i.e., $\rho_{0}$ and $\varphi_{0}$, as

$$
\begin{aligned}
& \left.\frac{\xi_{c}}{2} \operatorname{Im}\left(\boldsymbol{E}^{(0)} \cdot \boldsymbol{B}^{(0) *}\right)\right|_{\rho \rightarrow \text { rod's center }} \\
& \quad=p \xi_{c} Z_{\mathrm{TE}_{11}} \mu|C|^{2} \frac{\beta^{2}}{\left(\left(x_{11}^{\prime} / R\right)\right)^{3} \rho_{0}} J_{1}\left(\frac{x_{11}^{\prime}}{R} \rho_{0}\right) J_{1}\left(\frac{x_{11}^{\prime}}{R} \rho_{0}\right) .
\end{aligned}
$$

If the chiral rod is attached to the wall, $\rho_{0}=R$, and thus $\delta \beta / \beta$ due to chirality is nil since $J_{1}^{\prime}\left(x_{11}^{\prime}\right)=0$. If the chiral rod is placed at the center of the waveguide, i.e., $\rho_{0}=0$, and since the rod is assumed to be very small, (5) can be evaluated at $\rho_{0}=0$, resulting in $\left.\left(\xi_{c} / 2\right) \operatorname{Im}\left(\boldsymbol{E}^{(0)} \cdot B^{(0) *}\right)\right|_{\rho \rightarrow 0}=$ $p \xi_{\mathrm{c}} Z_{\mathrm{TE} \mathrm{T}_{11}} \mu|C|^{2} \cdot\left(\beta^{2} / 4\left(\left(x_{11}^{\prime} / R\right)\right)^{2}\right)$. Finally, we have the contribution of chirality to the relative change of the propagation constant due to insertion of the chiral rod, which can be expressed as

$$
\left.\frac{\delta \beta}{\beta}\right|_{c}=-4.18947 \cdot\left(\frac{d}{R}\right)^{2} \cdot \frac{p \xi_{c} Z_{\mathrm{TE}_{11}}}{1+p^{2}} .
$$

This relation reveals several important interesting effects of the chiral rod on the propagation constant. First, it shows that the relative change in $\beta$ is proportional to the local helicity of the unperturbed fields $p$ and the chirality admittance of the chiral rod $\xi_{c}$. If the handedness of the chiral medium (i.e., the sign of $\xi_{c}$ ) is reversed, the relative change in $\beta$ is reversed. Likewise, if the helicity of the unperturbed field is reversed, a similar effect occurs. When the fields are linearly polarized ( $p=0$ or $\infty$ ), the relative change due to chirality is nil. However, for RCP or LCP unperturbed fields where $p=+1$ or $-1, \delta \beta / \beta$ receives the maximum contribution from chirality. This suggests that a chiro-phase shifter would operate most effectively when there exists a right- (or left-) circularly polarized field at the rod's location in an otherwise unperturbed waveguide. This result was intuitively anticipated since the eigenmodes of propagation in chiral media are RCP 
and LCP waves, and chiral media interact with circularly polarized waves most effectively. It can also be observed from (5) [or (6)] that, if the direction of propagation is reversed (i.e., $\beta \rightarrow-\beta), Z_{\mathrm{TE}_{11}}$ will change its sign. Thus, in order to have the same $\delta \beta / \beta$, the sign of $p$ should change, which means that the helicity of the wave is reverse $d$. But since the direction of propagation is also changed, the handedness of the wave is unaltered. More explicitly, for example, unperturbed RCP fields would give rise to the same $\delta \beta / \beta$ regardless of the direction of propagation. This is not the case, however, for the magnetically biased ferrite [10]-[11]. This feature can, in principle, be used in the design of a reciprocal phase shifter using chiral materials. As shown above, the relative value of $\delta \beta / \beta$ depends on the helicity of the local unperturbed fields. For RCP and LCP fields, maximum interaction occurs between the chiral rod and the fields, while for linearly polarized fields, the chirality of the rod has no effect (to first order) on the value of the propagation constant. In the example given above, it was shown that unperturbed transverse fields can be chosen to be circularly polarized at the center of the guide. However, on the walls, these transverse fields will always be linearly polarized since at $\rho=a, H_{\rho}^{(0)}=0$ and $E_{\varphi}^{(0)}=0$. Therefore, if the chiral rod is on the walls, chirality will not contribute to the relative change in $\beta .^{5}$ This suggests that $\delta \beta / \beta$ is also affected by the location of the rod: when the rod is at the center of the guide, the effect of chirality on $\delta \beta / \beta$ is maximum (if the local fields are not linearly polarized), and as the rod moves towards the guide's walls, this effect is reduced. This feature can conceptually be used as a mechanical technique for achieving variable phase shifts. This can offer potential applications in the design of novel devices as displacement and pressure sensors for measuring mechanical displacement and pressure.

\section{SUMMARY}

Using a standard perturbation technique, we have discussed the effect of a thin chiral rod inserted longitudinally in a cylindrical metallic waveguide with arbitrary cross section on the propagation constant of guided modes. It has been found that in order to have a first-order effect due to the rod's chirality, the unperturbed fields $\boldsymbol{E}^{(0)}$ and $\boldsymbol{B}^{(0)}$ at the rod's location must provide a nonzero value for $\operatorname{Im}\left(\boldsymbol{E}^{(0)} \cdot \boldsymbol{B}^{(0) *}\right)$. A simple relation among the relative change in propagation constant and material parameters was obtained and given. This study has led us to suggest an idea for a novel phase shifter using chiral materials. This study has also shown that the phase shift can depend on the location of the chiral rod. This feature can potentially be used in the design of novel displacement sensors and for measuring chirality admittance.

\section{ACKNOWLEDGMENT}

We wish to thank the anonymous reviewers for their helpful comments and remarks on this paper, and in particular for

\footnotetext{
${ }^{5}$ Of course, as can be seen from (9), depending on the values of unperturbed fields at the rod's location, dielectric and magnetic properties of the rod may also contribute to $\delta \beta / \beta$.
}

bringing to our attention [12], [13], [18]. The work of [14] was submitted and appeared in print after the submission of our present paper. That work was brought to our attention while we were revising our manuscript. In that reference, the perturbation of the propagation constant of guided modes in a corrugated waveguide when a thin biisotropic rod is inserted is studied. In the problem studied here in our present paper, the waveguide is not corrugated, and the variation of the propagation constant is given in terms of the polarization of guided modes in a circular metallic waveguide.

\section{REFERENCES}

[1] J. Applequist, "Optical activity: Biot's bequest," Amer. Scientist, vol. 75 , no. 1, pp. 59-67, 1987.

[2] B. Lax and K. J. Button, Microwave Ferrites and Ferromagnetics. New York: McGraw-Hill, 1962.

[3] M. L. Kales, "Topics in guided-wave propagation in magnetized ferrites," Proc. IRE, vol. 44, pp. 1403-1409, 1956.

[4] A. A. T. M. Van Trier, "Guided electromagnetic waves in anisotropic media," Appl. Sci. Res., vol. 3B, pp. 305-371, 1953.

[5] D. L. Jaggard and N. Engheta, "Chirality in electrodynamics: Modeling and applications," in H. L. Bertoni and L. B. Felsen, Eds., Directions in Electromagnetic Wave Modelling. New York: Plenum, 1991.

[6] A. Lakhtakia, Ed., Selected Papers on Natural Optical Activity. Bellingham, WA: SPIE Opt. Eng. Press, 1990.

[7] A. Lakhtakia, V. V. Varadan, and V. K. Varadan, Time-Harmonic Electromagnetic Fields in Chiral Media. Berlin: Springer-Verlag, 1989.

[8] M. M. I. Saadoun and N. Engheta, "A reciprocal phase shifter using novel pseudochiral $\Omega$ medium," Microwave Opt. Tech.. Lett., vol. 5, no. 4, pp. 184-188, 1992.

[9] D. L. Jaggard, A. R. Mickelson, and C. H. Papas, "On electromagnetic waves in chiral media," Appl. Phys., vol. 18, pp. 211-216, 1979.

[10] R. A. Wadron, Theory of Guided Electromagnetic Waves. New York: Van Nostrand Reinhold, 1969.

[11] R. E. Collin, Field Theory of Guided Waves. New York: IEEE Press, 1991.

[12] A. Lakhtakia, "Perturbation of a cavity resonator by a small bianisotropic sphere," Int. J. Infrared Millimeter Waves, vol. 12, no. 2, pp. 109-114, 1991.

[13] S. Tretyakov and A. Viiatanen, "Perturbation theory for a cavity resonator with a biisotropic sample: Applications to measurement techniques," Microwave Opt. Tech. Lett., vol. 5, no. 4, pp. 174-177, 1992.

[14] A. Viitanen and I. V. Lindell, "Perturbation theory for a corrugated waveguide with a bi-isotropic rod," Microwave Opt. Tech. Lett., vol. 5, pp. 729-732, Dec. 1992.

[15] J. D. Jackson, Classical Electrodynamics. New York: Wiley, 1975.

[16] A. Lakhtakia, "Polarizability dyadics of small bianiostropic spheres," $J$. Phys. France, vol. 51, pp. 2235-2242, 1990.

[17] A. H. Sihvola, "Bi-isotropic mixtures," IEEE Trans. Antennas Propagat., vol. 40, pp. 188-197, Feb. 1992.

[18] J. Svedin, "Propagation analysis of chirowaveguides using the finiteelement method," IEEE Trans. Microwave Theory Tech., vol. 38, pp. 1488-1496, Oct. 1990.

[19] N. Engheta, D. L. Jaggard, and M. W. Kowarz, "Electromagnetic waves in Faraday chiral media," IEEE Trans. Antennas Propagat., vol. 40, pp. 367-374, Apr. 1992.

[20] T. D. Monte and P. L. E. Uslenghi, "Modal theory for a chiral-dielectric cable," Proc. 1990 IEEE AP-S Int. Symp./Radio Sci. Meet., Vol. Radio Sci. Meet., May 1990, p. 135.

Mamdouh M. I. Saadoun, photograph and biography, not available at the time of publication.

Nader Engheta (S'80-M'82-SM'89), photograph and biography, not available at the time of publication. 